\title{
LXIV. Theory of the telescopic level
}

\section{John Nixon Esq.}

To cite this article: John Nixon Esq. (1831) LXIV. Theory of the telescopic level, Philosophical Magazine Series 2, 9:54, 423-430, DOI: 10.1080/14786443108647651

To link to this article: http://dx.doi.org/10.1080/14786443108647651

曲 Published online: 27 Jul 2009.

Submit your article to this journal

Џ Article views: 2

Q View related articles $\sqsubset$ 
frequently attached a small barb of quill or fine iron, so as to assist more effectually in rendering extraction almost impossible. With the view of ensuring the arrow a straight course when ejected from the bow, they in common with all others who use the like instrument, attach a portion of feather to its hinder extremity, Of such, thus completed, every Bushman will perhaps be supplied with fifty or sixty, and those he carries in a sort of quiver, formed of the bark of the Kokkerboom, from which the woody part has been excavated. When, however, in a state of war, or in pursuit of game, he generally holds more or less loose in his hand, and when about to shoot, always places them in a convenient situation upon the ground.

The poison they employ is manufactured in various ways, so as to concentrate and render it adapted for application to the arrows. The most virulent sort, and that which they usually employ when they go against their enemies, is chiefly composed of the poison of snakes; the next to that is one obtained from the larvæ of an insect, found upon a bush growing near the Orange River; and the third is of vegetable origin, and called the malkop poison, on account of the peculiar effects it produces upon the senses. This last is not considered so serious in its consequences as either of the others, and is the sort commonly employed upon arrows destined for killing game.

Such then are a few of the points of interest connected with the history of the Bushmen; and though far from exhausting the subject, or even including all that my own notes would afford, yet $I$ am induced to conclude for the present, with an earnest recommendation to such of the members as may have been in the habit of observing our savage tribes, to embody their remarks for occasions like the present; as by such proceedings they may advance their individual reputations, at the same time that they acquire a consequence and character for our institution, which must be dear to all of us who feel a pride in the success of enterprizes in which we have a share.

\section{Theory of the Telescopic Level. By JoHn Nixon, Esq.*}

A TELESCOPIC level of the most simple construction, would consist of a refracting telescope with adjustable cross wires fixed within a perfectly cylindrical tube; the latter having attached to its surface (by means of adjusting screws) a spirit-level, placed parallel to the direction of its axis.

* Communicated by the Author. 
When in use, the telescope would rest within a trough formed by the junction of two planes (equally inclined to opposite points of the horizon, and secured to the surface of the upper parallel-plate of a tripod. In order to diminish the weight, without affecting the accuracy of the instrument, the artist makes a section of the trough, perpendicular to the line of junction of its sides, within a short distance of each end, and removes the whole of the intermediate part. These sections, or notches, (called Ys from their resemblance to that letter,) will be exactly opposite and parallel to each other, and have the same angular opening.

A spirit-level is a closed glass tube, nearly filled with spirits of wine, fixed to a straight bar, of which the under surface is a plane. A line drawn on this under surface in the direction of a plane passing through the axis of the tube, we shall call the reversing line of the level.

(For definitions of vertical, horizontal, and inclined lines and planes, see "'The Theory of the Spirit-level," Phil. Mag. and Annals, N.S. vol. i. p. 256.)

The reversing line of the level being placed, in opposite directions, in contact with a horizontal line, the bubble, considered as a point, will come to rest, in both instances, at the same point of the tube called the reversing point. A level may be placed in any direction in contact with a horizontal plane, without displacing the bubble (from its reversing point). When a level can be placed in contact with a plane in any two directions at right angles to each other, and reversed in both without deviation of the bubble, that plane is horizontal. When the reversing line of a level is placed in two opposite directions in contact with an inclined line, and the place of the bubble is marked in both instances on the tube, the distance between the two marks, converted into an arc of a circle, will be double the horizontal inclination of the line ;the reversing point lying between and equi-distant from the two marks. In a revolution of the level in contact with an inclined plane, the bubble would be twice at the (reversing or) same point of the tube; occurring when the reversing line of the level was in the direction, and in the opposite direction of one of the horizontal lines, which may be drawn (parallel to each other) through any point of an inclined plane;-and twice, when at right angles to these directions, the bubble would be at the same and maximum elongation from the stationary or reversing point, but on opposite sides of it; the equivalent angle of the linear space passed over by the bubble being the double of the horizontal inclination of the plane and line.-Parallel lines are either all inclined, or all parallel to the horizon. 
When any point of an object viewed through a refracting telescope is intercepted or apparently covered by the intersection of its cross-wires, that point of the object is in the direction of a straight line, termed the line of sight or collimation, passing through the intersecting point of the wires and the centre of refractions, or thickest part of the object-glass. When the telescope and wires are properly adjusted, the eye may be gradually withdrawn laterally from its original position in front of the eye-glass, without perceiving the least parallax, or deviation in the direction of the wires. Were wires, substituted for the object-glass, made to cross each other at the precise point previously occupied by its centre of refractions, we should find, on withdrawing the eye-tube, that a straight line passing through the intersection of the original and that of the substituted cross-wires (together forming what are called plain sights), would be in the direction of the same point of the object observed. The centre of the object-glass is known to coincide with that of its cell, and is consequently situated in, or very nearly in, the direction of the axis of the cylindrical tube, when the line of collimation, during a revolution of the glass within its cell, does not deviate from the object to which it was previously pointed. All the rays of light from a fixed star which enter a telescope are sensibly parallel to each other, but converge, after having passed through the object-glass, to a point (?) within the tube at a distance from the object-glass, called the sideral focus. Hence rays of light passing out of a telescope from the point of intersection of the wires, placed at the sideral focus, are inclined to each other, and diverge until they reach the object-glass, in which they suffer refraction, and emerge perfectly parallel to each other*. When two telescopes, properly adjusted with their cross-wires at the sideral focus, are placed nearly in a line, but in opposite directions, the wires of the further telescope, on looking through the nearer telescope, will appear perfectly distinct; whether the two object-glasses are in immediate contact or at a distance from each other. In either case, if the intersecting point of the wires of the nearer telescope is made to coincide with or intercept the view of the intersecting point of the wires of the further telescope, the lines of collimation of both telescopes will be strictly parallel to each othert. They cannot however be in one line (or direction), unless (- which we cannot ascertain (?)-) the centres of refraction of both object-glasses and the intersecting points of both cross-wires are all in the same straight line. Hence we

- Prof. Gauss of Goettingen.

N.S. Vol. 9. No. 54. June 1831.

+ Prof. Bessel of Konigsberg.

$3 \mathrm{I}$

may 
may move either telescope in a parallel clirection to a higher or lower level, or laterally; yet its wires, whilst visible, will still appear to cover at their intersecting point, that of the wires of the other telescope. Rays of light falling upon an object-glass from a distance within ten miles are sensibly divergent, and form their focus within the tube at a greater distance from the object-glass than the place of the sideral focus.

A straight line revolving about a parallel fixed line or axis at a constant distance from it, describes the longitudinal surface or sides of a cylinder. In the revolution of a cylinder about its fixed axis, every point of its sides describes a circle, to which that axis, passing through its centre, is perpendicular. The longitudinal lines, formed on the surface of the cylinder at any points of it, by planes passing in the direction of its axis, are parallel to that axis and to each other. When the sides of a cylinder press equally against a plane, the points of tangence form a straight line parallel to the axis of the cylinder. Sections of the cylinder by (parallel) planes passing perpendicular to its axis are circles, all of equal diameter, having the axis of the cylinder in the centre;-and the intersections by the same plane of the one in contact with the cylinder, will be as many parallel straight lines, tangents to, (and in the plane of) each corresponding circle of the section. A circle cannot come in contact with two lines inclined to each other, except at a point in each equally distant from the point in which they meet (the distance varying with the angular inclination of the lines). If we press a second plane, inclined to the first in an opposite direction, equally against the other side of the cylinder, transverse sections of the cylinder perpendicular to its axis, passing also through the two planes, will be circles, equal in diameter, in contact with two (tangential) lines inclined to each other at a constant angle, and the line of junction of the two planes will be parallel to the axis of the cylinder. A plane passing in the direction of the axis of the cylinder and that of the line of junction of the two planes will be equally inclined to both planes. The cylinder, in performing a revolution about its fixed axis, will aways be in contact with the same points of the two planes, which points form two lines parallel to the axis of rotation or that of the cylinder, and to themselves. When the cylinder is taken out of the trough formed by the two planes, and replaced within it reversed in direction, its axis must be situated in the same line as before, but in the opposite direction, because transverse sections of the cylinder and trough will still be circles of the previous diameter, in contact with two lines 
inclined to each other at the same angle as before. Consequently the cylinder, although reversed, is in contact with the trough at the former points, which lie in a line parallel to the axis of the cylinder and to the line of junction of the sides of the trough.

\section{Demonstration that the Line of Collimation of a perfect and well-adjusted Telescopic Level is truly horizontal.}

When properly adjusted, the position of the ends of the bubble relative to the level-tube being marked, the telescope (cylinder) may be taken out of its $\mathrm{Y}_{\mathrm{s}}$, and replaced, reversed in direction, yet the bubble will come to rest at the mark previously made on the tube. Also, on making the telescope perform an entire revolution within its $\mathrm{Ys}$, the point of intersection of its wires will continue to intercept the same point of the distant object observed.

Now as the line of collimation remains constant in direction during a revolution of the telescope within its Ys, it is evidently situated in or parallel to the axis of the cylinder, and is also parallel to either of the longitudinal lines on each side of the surface of the cylinder where in contact with the interior of the Ys, which latter are equivalent to the transverse sections of the two inclined planes or trough in contact with the cylinder.

Again : as the cylinder will suffer reversing within its $Y_{\mathbf{s}}$ without displacing the bubble, it follows that both these lines of contact are parallel to the horizon; and being also parallel to the line of collimation, the latter is perfectly horizontal.

Or, more simply: as the cylinder when reversed in the Ys has its axis in the same line as previously, and the reversing does not displace the bubble, the axis, and consequently the line of collimation, which is proved to be parallel to it, are horizontal.

In order to simplify the demonstration, the Ys have been considered as equal in angular opening. To prove that any inequality would not vitiate the instrument, we will furnish it with two additional $Y_{s}$, one wider, and the other narrower than the pair of equal Ys, in which the telescope, adjusted for observation, is supposed to rest. Our object being to place each additional $Y$ with both its sides in contact with the cylinder without disturbing the latter, we shall find it requisite to lower the narrower $\mathrm{Y}$, and raise the wider one; which will bring the one to touch the cylinder above, and the other below the level of the points of contact of the adjacent original Ys. Nevertheless, on reversing the cylinder, as it is of one diameter throughout its length, its contact with the four Ys 
will take place at points in each $Y$, and at perpendicular distances below its axis, the same as before reversing. Consequently we might remove the original $\mathrm{Ys}$, subsequent to reversing the telescope, without altering the place of the bubble.

It is also sufficiently evident, that when the $Y$ s are not exactly opposite to each other, (in which case they may be represented by sections of the trough oblique to the line of junction of its sides,) the cylinder will reverse within them precisely the same as though they were parallel.

The error of collimation of a telescopic level is the angle (measured on a vertical plane) expressing the inclination to the horizon of the line of sight or collimation. When the error is derived solely from imperfection in the instrument, it is termed constant or instrumental.

When the tube in which the telescope is placed is conical, or its ends are two cylinders of unequal diameter, the line of collimation, supposed level, will have a constant elevation or depression, accordingly as the object-glass is situated at the wider or narrower end of the tube.

Let us trace the consequences of increasing the diameter of, for instance, the object-glass end of the cylindrical tube of a perfect telescopic level adjusted for observation. In the first place, the thicker or object end of the tube will now come in contact with its $\mathrm{Y}$ above its former perpendicular height, without affecting the other or eye end; and the displaced bubble must come to rest at a point of its scale nearer to the object-glass. On reversing the tube, as the Ys are equal and their angular points are level with each other, neither the object nor the eye end of the tube suffer any consequent change of perpendicular height; the telescope is equally elevated after as before reversing, and the bubble must settle at its new mark.

The elevation indicated by the displacement of the bubble forms only a part of the error. When the tube was cylindrical, its axis would be at a perpendicular height above the angular point of either $\mathrm{Y}$, by a quantity equal to the secant of the complement of the angular opening of the $\mathrm{Y}$ multiplied by the radius of the cylinder. But in increasing the diameter of the object end of the tube, we have proportionately increased the height of its axis, at a point of it exactly over the $\mathrm{Y}$ in which it rests, whilst its height over the other $\mathrm{Y}$ may be considered as unaltered. Our conical tube will therefore reverse in equal Ys (of which the angular points are level with each other), without displacing the bubble (from its new position); yet the line of collimation (which may be adjusted to be fixed during 
a revolution of the tube within its $Y s$ ) will be constantly elevated at an angle of which the tangent is the (calculated) increase of perpendicular height divided by the distance between the Ys.

When the instrument is set up ready for taking levels, the upper surface of the tube* at the object end is at a greater elevation than at the eye end, by the difference of the semidiameters of the tube (measured over each $Y$ ), added to the corresponding augmentation of height of the axis. As the angles are minute, the horizontal inclination of the surface of the tube will be to that of its axis, (or to the error of collimation,) as the augmented perpendicular height of the former is to that of the latter. When the $Y_{s}$ open at 90 degrees, the ratio will be as $2 \cdot 414$ to $1 \cdot 414$.

In the case of the $\mathrm{Y}_{\mathrm{s}}$ being unequal, the error will be the mean of its value calculated for each $Y$. Supposing one of them $(X)$ to open at $90^{\circ}$, and the other $(Z)$ at $88^{\circ}$; and that the radius of the eye end (A) of the tube is 1.0 , and that of the object end (B) 1.2. When $A$ rests within $Z$, the height of the axis is 1.440 , and 1.697 with $B$ within $X$; so that it is more elevated over $X$ than over $Z$ by 0.257 . On reversing the tube, $A$ rests within $X$ at a height of $1 \cdot 414$, and $B$ within $Z$ at a height of $1 \cdot 728$; the axis being higher over $Z$ than over $X$ by 0.314 . But if we lower the narrower $\mathrm{Y}$ by 0.0285 (or half the difference), then will the elevation of the axis, before and after reversing, be equal to 0.4855 , or to the tangent of the constant error of collimation.

The following methods of ascertaining the instrumental error are the most feasible of those that have occurred to me. The result of each, on its application to determine the error of the horizon-sector, will be given hereafter.

I. Find by a spirit-level, placed on the surface of the tube, its inclination to the horizon.

II. Place a vessel filled with quicksilver between the objectglass of the instrument and that of another telescope $t$, and observe through the latter the intersection of the cross-wires of the former direct and by reflection.

III. By Captain Kater's horizontal floating Collimator.

IV. Make the lines of collimation of two telescopes parallel to that of the instrument, and afterwards point the telescopes at each other with the bubbles of their levels at their marks.

$\mathrm{V}$. Or measure by the instrument the minute inclination of

* Or, more correctly, of the line formed by the intersection of the upper surface of the tube by a vertical plane passing in the direction of its axis.

+ In every case the telescopes are understood to be adjusted to the sideral focus. 
the lines of collimation of two telescopes previously made parallel to each other.

VI. Compare two sets of observations, one of which was made before, and the other after the object-glass and eyepiece had been taken out of the instrument and replaced reversed in situation.

VII. Fix one spirit-level to the upper surface, and another to the under surface of an inflexible bar attached to the telescope. Make observations with the latter direct and inverted, and repeat them with the bar reversed in direction.

VIII. The error may be found by the double level affixed to the tube, without reference to the telescope.

IX. Were the bar of the double level moveable about a short horizontal axis projecting from either side of the tube, it might be inverted by making it describe exactly half a revolution. The two levels could then be made parallel to each other, and the reversing of the bar would be unnecessary.

$\mathrm{X}$. Place the instrument between two telescopes pointing at each other. Then make the line of collimation of the instrument parallel to that of either telescope, and reverse it within its Ys, which will bring its object-glass opposite to that of the other telescope. Having made the line of collimation of the latter parallel to that of the instrument, observe the difference of inclination, or deviation of parallelism of the lines of collimation of the two telescopes, the instrument being removed from between them.

XI. Take out the eye-tube and substitute an object-glass placed at a distance from the wires equal to its sideral focus. Point another telescope at, for instance, the original objectglass, and make their lines of collimation parallel. Reverse the instrument within its $\mathrm{Y}_{S}$, and note the deviation of parallelism of the lines of collimation of the additional object-glass and that of the (proof) telescope.

[To be continued.]

\section{Notices respecting Nerw Books.}

Illustrations of the Geology of Yorkshire; or a Description of the Strata and Organic Remains of the Yorkshire Coast: accompunied by a Geological Map, Sections, and Plates of the Fossil Plants and Animals. By Jonn Phiclips, F.G.S., Keeper of the Museum of the Yorkshire Philosophical Society, \&c. York, 1829. 4to. pp. 192. Twenty-four Lithographs.

[Concluded, from page 354.]

THE third chapter, containing descriptions of the Coast of Yorkshire from Spurn Point to Redcar, is illustrated by a coloured 